\title{
Existence and Global Uniform Asymptotic Stability of Almost Periodic Solutions for Cellular Neural Networks with Discrete and Distributed Delays
}

\author{
Zongyi Hou, ${ }^{1}$ Hongying Zhu, ${ }^{1}$ and Chunhua Feng ${ }^{2}$ \\ ${ }^{1}$ Department of Mathematics, Hechi University, Yizhou 546300, China \\ ${ }^{2}$ Department of Mathematics and Statistics, Guangxi Normal University, Guiling 541004, China \\ Correspondence should be addressed to Hongying Zhu; zhy71118@163.com
}

Received 29 September 2013; Revised 26 November 2013; Accepted 14 December 2013

Academic Editor: Samir Saker

Copyright (C) 2013 Zongyi Hou et al. This is an open access article distributed under the Creative Commons Attribution License, which permits unrestricted use, distribution, and reproduction in any medium, provided the original work is properly cited.

This paper discusses the existence and global uniform asymptotic stability of almost periodic solutions for cellular neural networks (CNNS). By utilizing the theory of the almost periodic differential equation and the Lyapunov functionals method, some sufficient conditions are obtained to ensure the existence and global uniform asymptotic stability. An example is given to illustrate the effectiveness of the main results.

\section{Introduction}

Cellular neural networks (CNNS) are composed of a large number of simple processing units (called neurons), widely interconnected to form a complex network system. It reflects many basic features of the human brain functions. It is a highly complicated nonlinear dynamics system and has successful applications in many fields such as associative, signal, and image processing, pattern recognition, and optimization.

In 1984, Hopfield proposed that the dynamic behavior of neurons should be described with a set of ordinary differential equations or functional differential equations. Since then, a lot of research achievements have been published in the world.

Recently, many scholars have paid much attention to the research on the dynamics and applications of CNNS. Specially, some scholars have studied the existence and stability of almost periodic solution for neural networks, which can be seen from [1-10] and therein references.

In [4], without product systems, by utilizing the generalized Halanay inequality technique and combining the theory of exponential dichotomy with fixed point method, Huang et al. study the existence and global exponential stability of almost periodic solutions for recurrent neural network with continuously distributed delays as follows:

$$
\begin{aligned}
x_{i}^{\prime}(t)= & -a_{i}(t) x_{i}(t)+\sum_{j=1}^{n} b_{i j}(t) f_{j}\left(x_{j}(t)\right) \\
& +\sum_{j=1}^{n} c_{i j}(t) g_{j}\left(x_{j}\left(t-\tau_{i j}(t)\right)\right) \\
& +\sum_{j=1}^{n} d_{i j}(t) \int_{-\infty}^{t} k_{i j}(t-s) h_{j}\left(x_{j}(s)\right) d s+I_{i}(t),
\end{aligned}
$$

where $f_{j}, g_{j}, h_{j}: R \rightarrow R, j=1,2, \ldots, n$ are incentive functions, which satisfy $f_{j}(0)=g_{j}(0)=h_{j}(0)=0 . a_{i}(t)$, $b_{i j}(t), c_{i j}(t)$ are all almost periodic functions.

In [5], Xiang and Cao discuss the following system:

$$
x_{i}^{\prime}(t)=-a_{i}\left(x_{i}(t)\right)+\sum_{j=1}^{n} b_{i j}(t) f_{j}\left(x_{j}(t)\right)
$$




$$
\begin{aligned}
& +\sum_{j=1}^{n} c_{i j}(t) g_{j}\left(x_{j}\left(t-\tau_{i j}(t)\right)\right) \\
& +\sum_{j=1}^{n} d_{i j}(t) \int_{-\infty}^{t} k_{i j}(t-s) h_{j}\left(x_{j}(s)\right) d s+I_{i}(t) .
\end{aligned}
$$

Without product systems, by using the Lyapunov functionals method and analytical skills, the results about the existence, attractivity, and exponential stability of almost periodic solutions for the system (2) are obtained.

However, a more general system than the systems above is discussed in this paper. We consider the existence and global uniform asymptotic stability of almost periodic solutions to the CNNS with discrete and continuously distributed delays. The system is as follows:

$$
\begin{aligned}
x_{i}^{\prime}(t)= & -a_{i}\left(t, x_{i}(t)\right) x_{i}(t)+\sum_{j=1}^{n} b_{i j}(t) f_{j}\left(x_{j}(t)\right) \\
& +\sum_{j=1}^{n} c_{i j}(t) g_{j}\left(x_{j}\left(t-\tau_{i j}\right)\right) \\
& +\sum_{j=1}^{n} d_{i j}(t) \int_{-\infty}^{t} k_{i j}(t-s) h_{j}\left(x_{j}(s)\right) d s+I_{i}(t) .
\end{aligned}
$$

By using Lemmas 3 and 4 in the next section and under the less restrictive conditions, some sufficient conditions are obtained to ensure the existence and global uniform asymptotic stability of almost periodic solutions to the system (3). An example is given to illustrate the effectiveness of the main results at last.

\section{Preliminaries}

In order to facilitate the following section description, we introduce some marks and basic definitions in this section.

If $f(t, \varphi)$ is an almost periodic in $t$ uniformly for $x \in \Omega$, where $\Omega \subseteq R^{n}$ is an open set, then the equation

$$
x^{\prime}(t)=f\left(t, x_{t}\right)
$$

is called lagging-type almost periodic differential equation. The following system is defined as the product systems of (4):

$$
x^{\prime}(t)=f\left(t, x_{t}(t)\right), \quad y^{\prime}(t)=f\left(t, y_{t}(t)\right) .
$$

Definition 1 (see [11]). If there is a constant $M=M(\sigma, \varphi)$ for $(\sigma, \varphi) \in \Omega \subseteq R \times C$ such that the solution $x(t, \sigma, \varphi)$ of (5) through $(\sigma, \varphi)$ when $t \geq \sigma-r$ satisfies $|x(t, \sigma, \varphi)|<M$, then the solution $x(t, \sigma, \varphi)$ is bound.

Definition 2 (see [11]). Lyapunov functionals $V(t, \varphi): R \times$ $C \rightarrow R, C=C\left([-r, 0], R^{n}\right)$. Suppose that the solution of (5) through $(\sigma, \varphi)$ is $x(t, \sigma, \varphi), x_{t}(\sigma, \varphi)$ is defined as $x(t+$ $\theta, \sigma, \varphi), \theta \in[-r, 0]$. The total derivative is defined as follows:

$$
\left.V^{\prime}(t, \varphi)\right|_{(5)}=\varlimsup_{h \rightarrow 0^{+}} \frac{1}{h}\left[V\left(t+h, x_{t+h}(t, \varphi)\right)-V(t, \varphi)\right] .
$$

Then $V_{(5)}^{\prime}(t, \varphi)$ is the right derivative of functionals $V(t, \varphi)$ along (5).
Lemma 3 (see [11]). Lagging-type almost periodic differential equation (5) has an asymptotically almost periodic solution $|\bar{x}(t)|$, which satisfies $|\bar{x}(t)| \leq a<H, H \in R$ or $H=+\infty$ for all $t \geq 0$ defined in $R_{+}$; then (5) has an almost periodic solution.

Lemma 4 (see [11]). There is a continuous $V$ functional of $V(t, \varphi, \psi)$ for $t \geq 0, \varphi, \psi \in C_{H}, C_{H}=\{\varphi: \varphi \in C,|\varphi|<H\}$, $C=C[-r, 0],|\varphi|=\sup _{\theta \in[-r, 0]}|\varphi(\theta)|=\sup _{\theta \in[-r, 0]} \sum_{i=1}^{n}\left|\varphi_{i}(\theta)\right|$ such that

(Ha) $u(|\varphi-\psi|) \leq V(t, \varphi, \psi) \leq v(|\varphi-\psi|)$,

(Hb) $\left|V\left(t, \varphi_{1}, \psi_{1}\right)-V\left(t, \varphi_{2}, \psi_{2}\right)\right| \leq k\left(\left|\varphi_{1}-\varphi_{2}\right|+\left|\psi_{1}-\psi_{2}\right|\right)$,

(Hc) $V_{(5)}^{\prime}(t, \varphi, \psi) \leq-a V(t, \varphi, \psi)$,

where $a$ is a positive constant, $u(s)$ and $v(s)$ are continuous and non-decreasing, when $s \rightarrow 0, u(s) \rightarrow 0$, and $k$ is a positive constant. At this time, if (5) has a bounded solution $x(t, \sigma, \varphi)$ such that $|x(t, \sigma, \varphi)| \leq H_{1}$, where $t \geq \sigma \geq 0, H>H_{1}>0$, then (5) in $C_{H}$ has a unique almost periodic solution which is global uniform asymptotic stability. tions.

Throughout this paper, we make the following assump-

(H2.1) $a_{i}\left(t, x_{i}\right)$ is uniform almost periodic continuous function to $x_{i}$ about $t . a_{i}\left(t, x_{i}\right)>0$ for all $\left(t, x_{i}\right)$, and we denote $\min _{1 \leq i \leq n}\left\{\inf _{\left(t, x_{i}\right) \in R \times R} a_{i}\left(t, x_{i}\right)\right\}=2 a_{0}>0$. In addition, $a_{i}\left(t, x_{i}\right)$ also satisfies the Lipschitz condition as follows:

$$
\left|a_{i}\left(t, x_{i}\right)-a_{i}\left(t, y_{i}\right)\right| \leq L_{i}^{a}\left|x_{i}-y_{i}\right|
$$

where $L_{i}^{a}<a_{0}$.

$(\mathrm{H} 2.2) b_{i j}(t), c_{i j}(t), d_{i j}(t)$ and $I_{i}(t)$ are almost periodic continuous functions. we denote constants, respectively, $b_{i j}^{l}, b_{i j}^{m}, c_{i j}^{l}, c_{i j}^{m}, d_{i j}^{l}, d_{i j}^{m}, I_{i}^{l}, I_{i}^{m}(i, j=1,2, \ldots, n)$ as follows:

$$
\begin{array}{cl}
\inf _{t \in R} b_{i j}(t)=b_{i j}^{l}, & \sup _{t \in R} b_{i j}(t)=b_{i j}^{m}>0, \\
\inf _{t \in R} c_{i j}(t)=c_{i j}^{l}, & \sup _{t \in R} c_{i j}(t)=c_{i j}^{m}>0, \\
\inf _{t \in R} d_{i j}(t)=d_{i j}^{l}, & \sup _{t \in R} d_{i j}(t)=d_{i j}^{m}>0, \\
\inf _{t \in R} I_{i}(t)=I_{i}^{l}, & \sup _{t \in R} I_{i}(t)=I_{i}^{m}>0 .
\end{array}
$$

(H2.3) Functions $f_{j}\left(x_{j}\right), g_{j}\left(x_{j}\right)$, and $h_{j}\left(x_{j}\right)$ are bounded continuous functions, and they satisfy the following Lipschitz conditions:

$$
\begin{aligned}
& \left|f_{j}\left(x_{j}\right)-f_{j}\left(y_{j}\right)\right| \leq L_{j}^{f}\left|x_{j}-y_{j}\right|, \\
& \left|g_{j}\left(x_{j}\right)-g_{j}\left(y_{j}\right)\right| \leq L_{j}^{g}\left|x_{j}-y_{j}\right|, \\
& \left|h_{j}\left(x_{j}\right)-h_{j}\left(y_{j}\right)\right| \leq L_{j}^{h}\left|x_{j}-y_{j}\right| .
\end{aligned}
$$


(H2.4) Delay kernel functions satisfying

$$
\begin{array}{r}
\int_{0}^{\infty} k_{i j}(s) d s=1, \quad \int_{0}^{\infty} s k_{i j}(s) d s<+\infty \\
(i, j=1,2, \ldots, n) .
\end{array}
$$

\section{Main Results}

Theorem 5. Assume that (H2.1)-(H2.4) hold; then all solutions of system (3) are bounded.

Proof. Let $\left|f_{j}\left(x_{j}\right)\right| \leq P_{j},\left|g_{j}\left(x_{j}\right)\right| \leq Q_{j},\left|h_{j}\left(x_{j}\right)\right| \leq R_{j}$ and set $N=\sum_{j=1}^{n} b_{i j}^{m} P_{j}+\sum_{j=1}^{n} c_{i j}^{m} Q_{j}+\sum_{j=1}^{n} d_{i j}^{m} R_{j}+I_{i}^{m}$. From system (3) and the assumption (H2.3), we get

$$
\begin{aligned}
x_{i}^{\prime}(t)= & -a_{i}\left(t, x_{i}(t)\right) x_{i}(t)+\sum_{j=1}^{n} b_{i j}(t) f_{j}\left(x_{j}(t)\right) \\
& +\sum_{j=1}^{n} c_{i j}(t) g_{j}\left(x_{j}\left(t-\tau_{i j}\right)\right) \\
& +\sum_{j=1}^{n} d_{i j}(t) \int_{-\infty}^{t} k_{i j}(t-s) h_{j}\left(x_{j}(s)\right) d s+I_{i}(t) \\
\leq & -a_{i}\left(t, x_{i}(t)\right) x_{i}(t)+\sum_{j=1}^{n} b_{i j}^{m} P_{j} \\
& +\sum_{j=1}^{n} c_{i j}^{m} Q_{j}+\sum_{j=1}^{n} d_{i j}^{m} R_{j}+I_{i}^{m} \\
= & -a_{i}\left(t, x_{i}(t)\right) x_{i}(t)+N,
\end{aligned}
$$

This shows that

$$
\left|x_{i}(t)\right| \leq \frac{N}{2 a_{0}}, \quad i=1,2, \ldots, n .
$$

This completes the proof of the theorem.

From Theorem 5, all solutions of system (3) are bounded. In order to investigate the globally uniform asymptotic stability of the almost periodic solutions, we assume that $\sum_{i=1}^{n}\left(N / 2 a_{0}\right) L_{i}^{a} \leq a_{0}$.

Theorem 6. Assume that (H2.1)-(H2.4) hold, and suppose further that

$$
\sigma=\min _{1 \leq i \leq n}\left\{a_{0}-\sum_{j=1}^{n} b_{i j}^{m} L_{i}^{f}-\sum_{j=1}^{n} c_{i j}^{m} L_{j}^{g}-\sum_{j=1}^{n} d_{i j}^{m} L_{j}^{h}\right\}>0 .
$$

Then, in system (3), there exists an almost periodic solution, which is global uniform asymptotic stable.

Proof. From the condition (H2.1), we rewrite system (3) as follows:

$$
\begin{aligned}
x_{i}^{\prime}(t)= & -a_{i}\left(t, x_{i}(t)\right) x_{i}(t)+\sum_{j=1}^{n} b_{i j}(t) f_{j}\left(x_{j}(t)\right) \\
& +\sum_{j=1}^{n} c_{i j}(t) g_{j}\left(x_{j}\left(t-\tau_{i j}\right)\right) \\
& +\sum_{j=1}^{n} d_{i j}(t) \int_{-\infty}^{t} k_{i j}(t-s) h_{j}\left(x_{j}(s)\right) d s+I_{i}(t) .
\end{aligned}
$$

The product system of the system (15) is the following form:

$$
\begin{aligned}
x_{i}^{\prime}(t)= & -a_{i}\left(t, x_{i}(t)\right) x_{i}(t)+\sum_{j=1}^{n} b_{i j}(t) f_{j}\left(x_{j}(t)\right) \\
& +\sum_{j=1}^{n} c_{i j}(t) g_{j}\left(x_{j}\left(t-\tau_{i j}\right)\right) \\
& +\sum_{j=1}^{n} d_{i j}(t) \int_{-\infty}^{t} k_{i j}(t-s) h_{j}\left(x_{j}(s)\right) d s+I_{i}(t), \\
y_{i}^{\prime}(t)= & -a_{i}\left(t, y_{i}(t)\right) y_{i}(t)+\sum_{j=1}^{n} b_{i j}(t) f_{j}\left(y_{j}(t)\right) \\
& +\sum_{j=1}^{n} c_{i j}(t) g_{j}\left(y_{j}\left(t-\tau_{i j}\right)\right) \\
& +\sum_{j=1}^{n} d_{i j}(t) \int_{-\infty}^{t} k_{i j}(t-s) h_{j}\left(y_{j}(s)\right) d s+I_{i}(t) .
\end{aligned}
$$

In order to apply Lemma 4, we construct the Lyapunov functionals about the product system (16) as follows:

$$
\begin{aligned}
V(t, x(\cdot), y(\cdot))= & \sum_{i=1}^{n}\left|x_{i}(t)-y_{i}(t)\right| \\
& +\sum_{i=1}^{n} \sum_{j=1}^{n} c_{i j}^{m} L_{j}^{g} \int_{t-\tau_{i j}}^{t}\left|x_{j}(s)-y_{j}(s)\right| d s \\
& +\sum_{i=1}^{n} \sum_{j=1}^{n} d_{i j}^{m} L_{j}^{h} \\
& \times \int_{0}^{+\infty} k_{i j}(s) \int_{t-s}^{t}\left|x_{j}(u)-y_{j}(u)\right| d u d s .
\end{aligned}
$$

For convenience sake, we denote

$$
V(t, x(\cdot), y(\cdot))=V_{1}(t)+V_{2}(t)+V_{3}(t),
$$


where

$$
\begin{gathered}
V_{1}(t)=\sum_{i=1}^{n}\left|x_{i}(t)-y_{i}(t)\right| \\
V_{2}(t)=\sum_{i=1}^{n} \sum_{j=1}^{n} c_{i j}^{m} L_{j}^{g} \int_{t-\tau_{i j}}^{t}\left|x_{j}(s)-y_{j}(s)\right| d s \\
V_{3}(t)=\sum_{i=1}^{n} \sum_{j=1}^{n} d_{i j}^{m} L_{j}^{h} \int_{0}^{+\infty} k_{i j}(s) \int_{t-s}^{t}\left|x_{j}(u)-y_{j}(u)\right| d u d s .
\end{gathered}
$$

Using (H2.1) and $\sum_{i=1}^{n}\left(N / 2 a_{0}\right) L_{i}^{a} \leq a_{0}$, by the triangle inequality, we have

$$
\begin{aligned}
\operatorname{sgn} \sum_{i=1}^{n}\left(-a_{i}\left(t, x_{i}(t)\right) x_{i}(t)+a_{i}\left(t, y_{i}(t)\right) y_{i}(t)\right) \\
=\operatorname{sgn} \sum_{i=1}^{n}\left\{-a_{i}\left(t, x_{i}(t)\right) x_{i}(t)+a_{i}\left(t, y_{i}(t)\right) x_{i}(t)\right. \\
\left.\quad-a_{i}\left(t, y_{i}(t)\right) x_{i}(t)+a_{i}\left(t, y_{i}(t)\right) y_{i}(t)\right\} \\
\leq \operatorname{sgn} \sum_{i=1}^{n}\left\{\left(x_{i}(t)-y_{i}(t)\right) \frac{N}{2 a_{0}} L_{i}^{a}-2 a_{0}\left(x_{i}(t)-y_{i}(t)\right)\right\} \\
\leq-a_{0} \operatorname{sgn} \sum_{i=1}^{n}\left(x_{i}(t)-y_{i}(t)\right) .
\end{aligned}
$$

Calculating the upright derivative of $V_{1}(t)$ along system (16) as follows:

$$
\begin{aligned}
& \left.D^{+} V_{1}(t)\right|_{(16)} \\
& \leq-a_{0} \sum_{i=1}^{n}\left|x_{i}(t)-y_{i}(t)\right| \\
& \quad+\sum_{i=1}^{n} \sum_{j=1}^{n}\left|b_{i j}(t)\right|\left|f_{j}\left(x_{j}(t)\right)-f_{j}\left(y_{j}(t)\right)\right| \\
& \quad+\sum_{i=1}^{n} \sum_{j=1}^{n}\left|c_{i j}(t)\right|\left|g_{j}\left(x_{j}\left(t-\tau_{i j}\right)\right)-g_{j}\left(x_{j}\left(t-\tau_{i j}\right)\right)\right| \\
& \quad+\sum_{i=1}^{n} \sum_{j=1}^{n}\left|d_{i j}(t)\right| \int_{-\infty}^{t} k_{i j}(t-s)\left|h_{j}\left(x_{j}(s)\right)-h_{j}\left(y_{j}(s)\right)\right| d s .
\end{aligned}
$$

Similarly, we calculate the upright derivatives of $V_{2}(t)$ and $V_{3}(t)$ along system (16), respectively, as follows:

$$
\begin{aligned}
& \left.D^{+} V_{2}(t)\right|_{(16)} \\
& =\sum_{i=1}^{n} \sum_{j=1}^{n} c_{i j}^{m} L_{j}^{g}\left|x_{j}(t)-y_{j}(t)\right| \\
& \quad-\sum_{i=1}^{n} \sum_{j=1}^{n} c_{i j}^{m} L_{j}^{g}\left|x_{j}\left(t-\tau_{i j}\right)-y_{j}\left(t-\tau_{i j}\right)\right| ;
\end{aligned}
$$

$$
\begin{aligned}
\left.D^{+} V_{3}(t)\right|_{(16)} & \\
= & \sum_{i=1}^{n} \sum_{j=1}^{n} d_{i j}^{m} L_{j}^{h} \int_{0}^{+\infty} k_{i j}(s)\left|x_{j}(t)-y_{j}(t)\right| d s \\
& \quad-\sum_{i=1}^{n} \sum_{j=1}^{n} d_{i j}^{m} L_{j}^{h} \int_{0}^{+\infty} k_{i j}(s)\left|x_{j}(t-s)-y_{j}(t-s)\right| d s .
\end{aligned}
$$

Note that

$$
\begin{aligned}
& \sum_{i=1}^{n} \sum_{j=1}^{n}\left|c_{i j}(t)\right|\left|g_{j}\left(x_{j}\left(t-\tau_{i j}\right)\right)-g_{j}\left(x_{j}\left(t-\tau_{i j}\right)\right)\right| \\
& \quad \leq \sum_{i=1}^{n} \sum_{j=1}^{n} c_{i j}^{m} L_{j}^{g}\left|x_{j}\left(t-\tau_{i j}\right)-y_{j}\left(t-\tau_{i j}\right)\right|, \\
& \sum_{i=1}^{n} \sum_{j=1}^{n}\left|d_{i j}(t)\right| \int_{-\infty}^{t} k_{i j}(t-s)\left|h_{j}\left(x_{j}(s)\right)-h_{j}\left(y_{j}(s)\right)\right| d s \\
& \quad \leq \sum_{i=1}^{n} \sum_{j=1}^{n} d_{i j}^{m} L_{j}^{h} \int_{0}^{+\infty} k_{i j}(s)\left|x_{j}(t)-y_{j}(t)\right| d s .
\end{aligned}
$$

Combining with (21) and (22) and the assumptions of Theorem 5, we get

$$
\begin{aligned}
D^{+} V & (t, x(\cdot), y(\cdot))||_{(16)} \\
\leq & -a_{0} \sum_{i=1}^{n}\left|x_{i}(t)-y_{i}(t)\right| \\
& +\sum_{i=1}^{n} \sum_{j=1}^{n}\left|b_{i j}(t)\right|\left|f_{j}\left(x_{j}(t)\right)-f_{j}\left(y_{j}(t)\right)\right| \\
& +\sum_{i=1}^{n} \sum_{j=1}^{n}\left|c_{i j}(t)\right|\left|g_{j}\left(x_{j}\left(t-\tau_{i j}\right)\right)-g_{j}\left(x_{j}\left(t-\tau_{i j}\right)\right)\right| \\
& +\sum_{i=1}^{n} \sum_{j=1}^{n}\left|d_{i j}(t)\right| \int_{-\infty}^{t} k_{i j}(t-s) \\
& +\sum_{i=1}^{n} \sum_{j=1}^{n} c_{i j}^{m} L_{j}^{g}\left|x_{j}(t)-y_{j}(t)\right| \\
& -\sum_{i=1}^{n} \sum_{j=1}^{n} c_{i j}^{m} L_{j}^{g}\left|x_{j}\left(t-\tau_{i j}\right)-y_{j}\left(t-\tau_{i j}\right)\right| \\
& +\sum_{i=1}^{n} \sum_{j=1}^{n} d_{i j}^{m} L_{j}^{h} \int_{0}^{+\infty} k_{i j}(s)\left|x_{j}(t)-y_{j}(t)\right| d s \\
& -\sum_{i=1}^{n} \sum_{j=1}^{n} d_{i j}^{m} L_{j}^{h} \int_{0}^{+\infty} k_{i j}(s)\left|x_{j}(t-s)-y_{j}(t-s)\right| d s \\
&
\end{aligned}
$$




$$
\begin{aligned}
\leq & -a_{0} \sum_{i=1}^{n}\left|x_{i}(t)-y_{i}(t)\right| \\
& +\sum_{i=1}^{n} \sum_{j=1}^{n}\left|b_{i j}(t)\right|\left|f_{j}\left(x_{j}(t)\right)-f_{j}\left(y_{j}(t)\right)\right| \\
& +\sum_{i=1}^{n} \sum_{j=1}^{n} c_{i j}^{m} L_{j}^{g}\left|x_{j}(t)-y_{j}(t)\right| \\
& +\sum_{i=1}^{n} \sum_{j=1}^{n} d_{i j}^{m} L_{j}^{h} \int_{0}^{+\infty} k_{i j}(s)\left|x_{j}(t)-y_{j}(t)\right| d s \\
\leq & -a_{0} \sum_{i=1}^{n}\left|x_{i}(t)-y_{i}(t)\right|+\sum_{i=1}^{n} \sum_{j=1}^{n} b_{i j}^{m} L_{j}^{f}\left|x_{j}(t)-y_{j}(t)\right| \\
& +\sum_{i=1}^{n} \sum_{j=1}^{n} c_{i j}^{m} L_{j}^{g}\left|x_{j}(t)-y_{j}(t)\right| \\
& +\sum_{i=1}^{n} \sum_{j=1}^{n} d_{i j}^{m} L_{j}^{h}\left|x_{j}(t)-y_{j}(t)\right| \\
\leq & -\sigma \sum_{i=1}^{n}\left|x_{i}(t)-y_{i}(t)\right| . \\
\leq & -a_{0} \sum_{i=1}^{n}\left|x_{i}(t)-y_{i}(t)\right|+\sum_{i=1}^{n} \sum_{j=1}^{n} b_{j i}^{m} L_{i}^{f}\left|x_{i}(t)-y_{j}(t)\right| \\
& \left.\times c_{j i}^{m} L_{i}^{g}\left|x_{i}(t)-y_{i}(t)\right|+\sum_{i=1}^{n} \sum_{j=1}^{n} d_{j i}^{m} L_{i}^{m} L_{i}^{f} \mid x_{i}(t)-\sum_{j=1}^{n} c_{j i}^{m} L_{i}^{g}+\sum_{j=1}^{n} d_{j i}^{m} L_{i}^{h}\right\} \\
& \\
&
\end{aligned}
$$

By Theorem 5 and Lemmas 3 and 4, there exists an almost periodic solution of system (3), which is global uniform asymptotic stable. This completes the proof of Theorem 6 .

\section{An Example}

Example 1. Consider the following cellular neural network which consists of two neurons:

$$
\begin{aligned}
x_{i}^{\prime}(t)= & -a_{i}\left(t, x_{i}(t)\right) x_{i}(t)+\sum_{j=1}^{n} b_{i j}(t) f_{j}\left(x_{j}(t)\right) \\
& +\sum_{j=1}^{n} c_{i j}(t) g_{j}\left(x_{j}\left(t-\tau_{i j}\right)\right)
\end{aligned}
$$

$$
\begin{array}{r}
+\sum_{j=1}^{n} d_{i j}(t) \int_{-\infty}^{t} k_{i j}(t-s) h_{j}\left(x_{j}(s)\right) d s+I_{i}(t), \\
i=1,2,
\end{array}
$$

where

$$
\begin{aligned}
& \left(a_{i}\left(t, x_{i}(t)\right) x_{i}(t)\right)_{2 \times 2} \\
& =\left(\begin{array}{cc}
\frac{11-e^{-\left|x_{1}(t)\right|}+\sin \pi t}{2-\sin x_{2}(t)} x_{1}(t) & 0 \\
0 & \frac{11-e^{-\left|x_{2}(t)\right|}-\cos t}{2+\sin x_{1}(t)} x_{2}(t)
\end{array}\right), \\
& \left(b_{i j}(t)\right)_{2 \times 2}=\left(\begin{array}{cc}
0.2 \sin \pi t & 0.1 \cos t \\
0.1 \sin t & 0.2 \cos \pi t
\end{array}\right), \\
& \left(c_{i j}(t)\right)_{2 \times 2}=\left(\begin{array}{cc}
0.2 \sin t & 0.1 \sin t \\
0.2 \sin 2 t & 0.1 \cos \pi t
\end{array}\right), \\
& \left(d_{i j}(t)\right)_{2 \times 2}=\left(\begin{array}{cc}
0.3 \sin 2 t & 0.1 \cos t \\
0.2 \sin \pi t & 0.1 \cos \pi t
\end{array}\right), \\
& \left(I_{i}(t)\right)_{1 \times 2}=\left(\begin{array}{cc}
0.1 \sin 2 t \\
0.1 \cos t
\end{array}\right) .
\end{aligned}
$$

We select the functions $f_{j}(x)=g_{j}(x)=h_{j}(x)=$ $\operatorname{sh}(x) / \operatorname{ch}(x)$ and the kernel functions $k_{i j}(s)=e^{-s}$. Then, $L_{j}^{f}=L_{j}^{g}=L_{j}^{h}=1$. Because the periods of $\sin \pi t$ and $\cos t$ are 2 and $2 \pi$, respectively. The quotient of $2 \pi$ and 2 is irrational. Then system (25) is an almost periodic system. In addition, $a_{0}=1.5$ and

$$
\sum_{j=1}^{2} b_{i j}^{m} L_{i}^{f}+\sum_{j=1}^{2} c_{i j}^{m} L_{j}^{g}+\sum_{j=1}^{2} d_{i j}^{m} L_{j}^{h} \leq 1.2<1.5 .
$$

From $P_{j}=Q_{j}=R_{j}=1$, we have $N=\sum_{j=1}^{n} b_{i j}^{m} P_{j}+\sum_{j=1}^{n} c_{i j}^{m} Q_{j}+$ $\sum_{j=1}^{n} d_{i j}^{m} R_{j}+I_{i}^{m} \leq 1.2<a_{0}$; then we get $\sum_{i=1}^{n}\left(N / 2 a_{0}\right) L_{i}^{a}=$ $0.2<1.5$.

It is easy for us to verify that the conditions (H2.1)(H2.4) in Theorem 5 hold. Therefore, in the system (25), there exists an almost periodic solution, which is global uniform asymptotic stable.

\section{Acknowledgments}

This work was supported by the National Natural Science Foundation of China (11361010 and 11161018); the Institutions of Higher Learning in Guangxi of China, Scientific Research Fund Project (201204LX391); the Scientific Research Project in Guangxi of China, Department of Education (201106LX613).

\section{References}

[1] C. Bai, "Existence and stability of almost periodic solutions of Hopfield neural networks with continuously distributed delays," Nonlinear Analysis: Theory, Methods \& Applications A, vol. 71, no. 11, pp. 5850-5859, 2009. 
[2] J. Cao, A. Chen, and X. Huang, "Almost periodic attractor of delayed neural networks with variable coefficients," Physics Letters A, vol. 340, no. 2, pp. 104-120, 2005.

[3] A. Chen and J. Cao, "Existence and attractivity of almost periodic solutions for cellular neural networks with distributed delays and variable coefficients," Applied Mathematics and Computation, vol. 134, no. 1, pp. 125-140, 2003.

[4] X. Huang, J. Cao, and D. W. C. Ho, "Existence and attractivity of almost periodic solution for recurrent neural networks with unbounded delays and variable coefficients," Nonlinear Dynamics, vol. 45, no. 3-4, pp. 337-351, 2006.

[5] H. Xiang and J. Cao, "Almost periodic solutions of recurrent neural networks with continuously distributed delays," Nonlinear Analysis: Theory, Methods \& Applications A, vol. 71, no. 12, pp. 6097-6108, 2009.

[6] B. Liu and L. Huang, "Positive almost periodic solutions for recurrent neural networks," Nonlinear Analysis: Real World Applications, vol. 9, no. 3, pp. 830-841, 2008.

[7] M. Pinto and G. Robledo, "Existence and stability of almost periodic solutions in impulsive neural network models," Applied Mathematics and Computation, vol. 217, no. 8, pp. 4167-4177, 2010.

[8] S. Qin, X. Xue, and P. Wang, "Global exponential stability of almost periodic solution of delayed neural networks with discontinuous activations," Information Sciences, vol. 220, no. 20, pp. 367-378, 2013.

[9] L. Wang, "Existence and global attractivity of almost periodic solutions for delayed high-ordered neural networks," Neurocomputing, vol. 73, no. 4-6, pp. 802-808, 2010.

[10] J. Zhou, W. Zhao, X. Lv, and H. Zhu, "Stability analysis of almost periodic solutions for delayed neural networks without global Lipschitz activation functions," Mathematics and Computers in Simulation, vol. 81, no. 11, pp. 2440-2455, 2011.

[11] Z. X. Zheng, Theory of Functional Differential Equation, Anhui Education Publishing Press, 1994. 


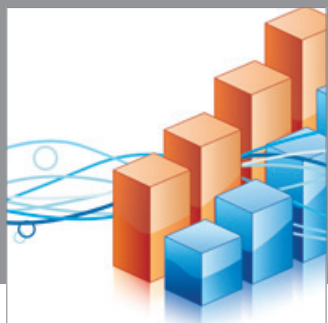

Advances in

Operations Research

mansans

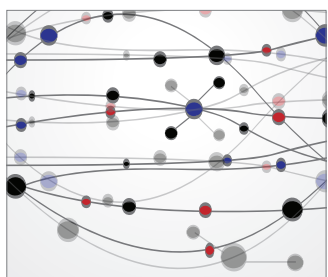

The Scientific World Journal
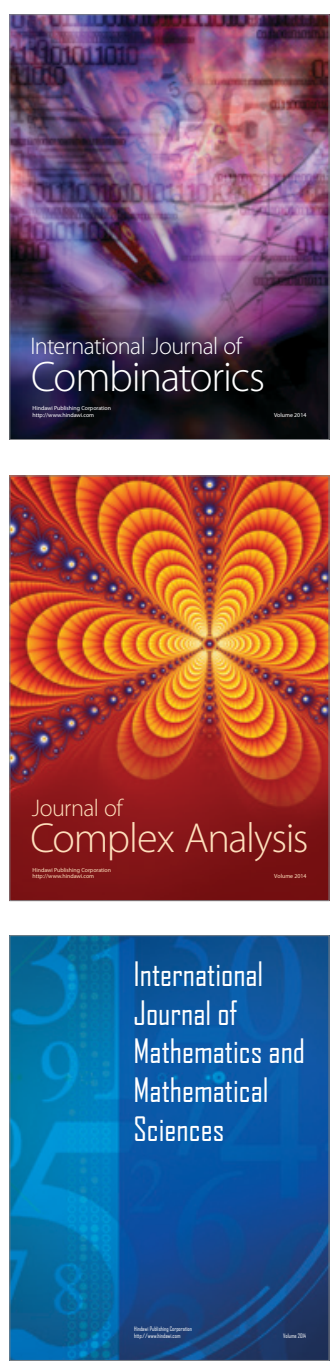
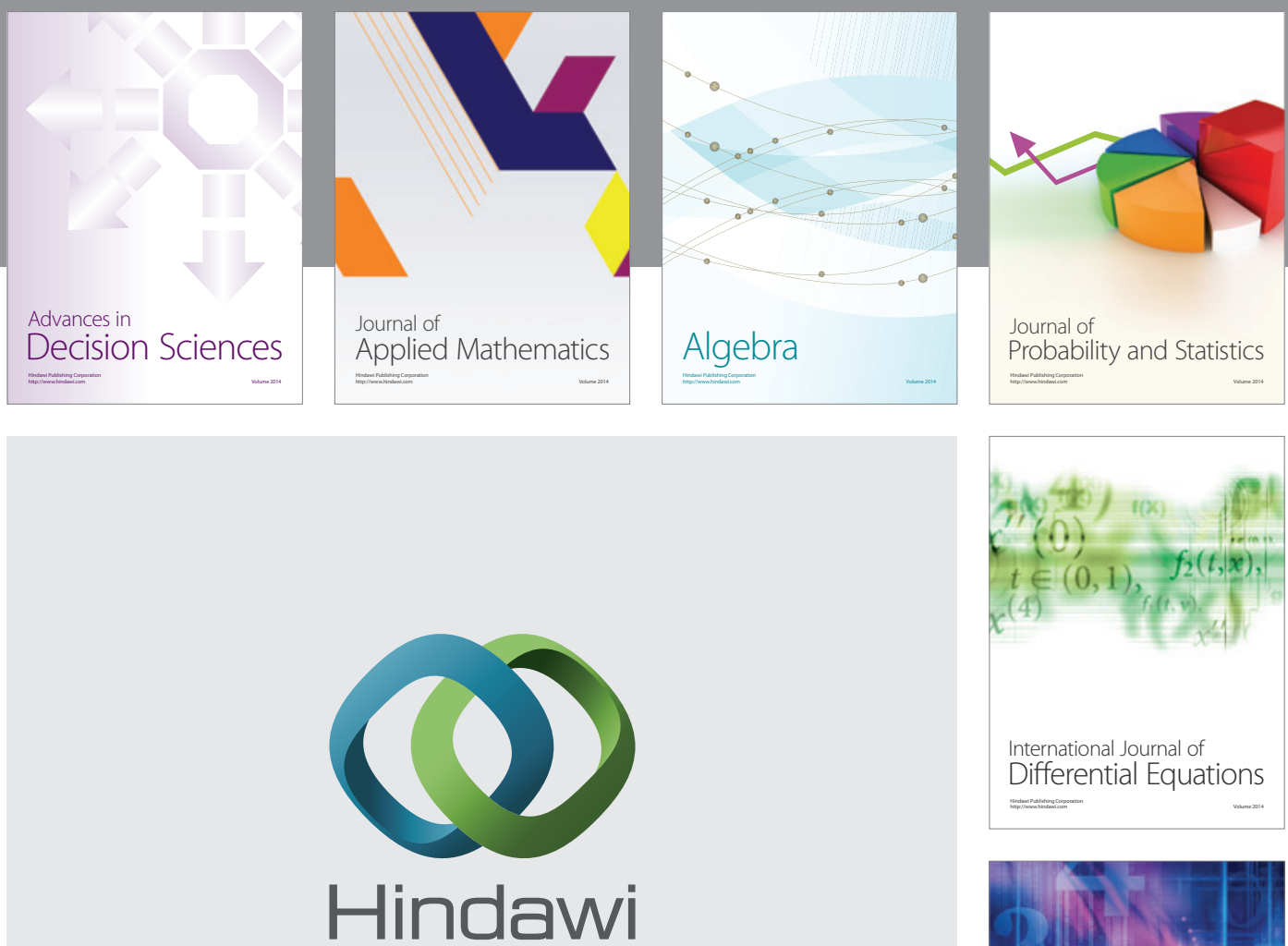

Submit your manuscripts at http://www.hindawi.com
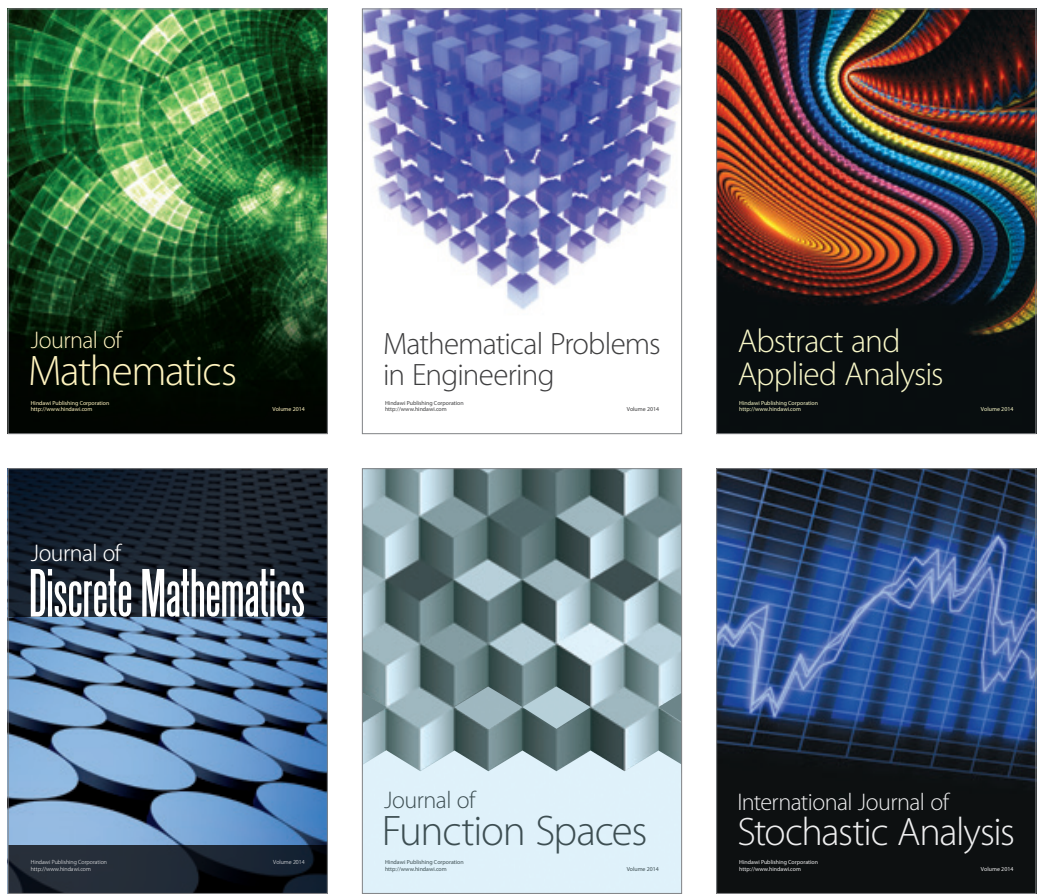

Journal of

Function Spaces

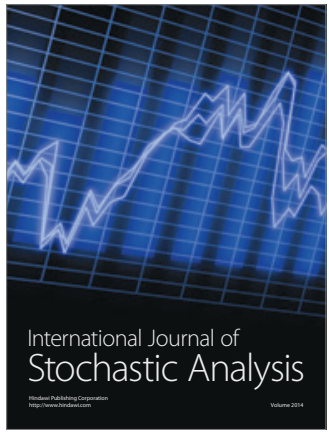

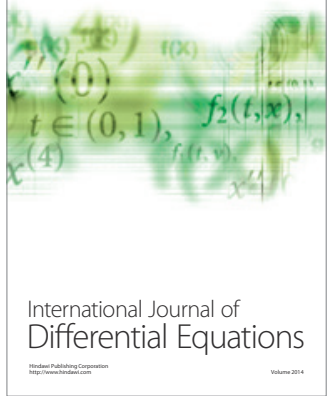
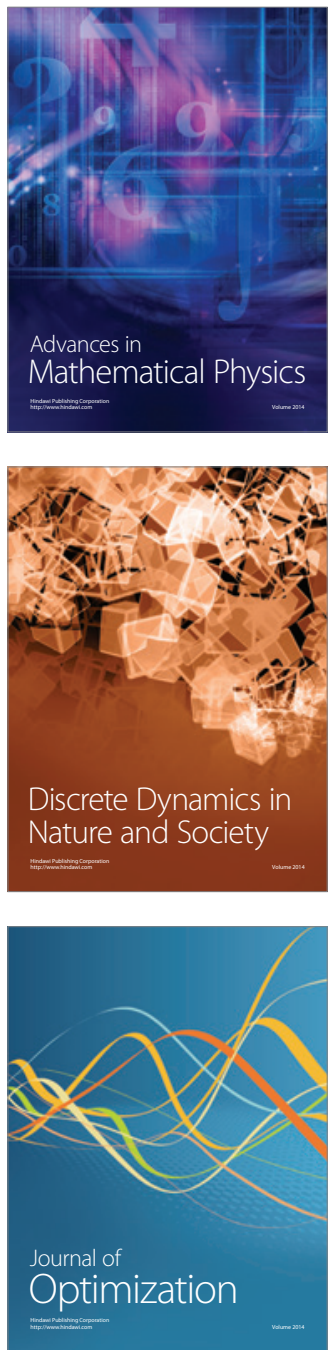